\title{
Mid-Infrared Silicon Photonics
}

\author{
William M. J. Green ${ }^{1}$, Bart Kuyken ${ }^{2}$, Xiaoping Liu ${ }^{3}$, Mackenzie A. Van Camp ${ }^{1}$, S. Assefa ${ }^{1}$, Douglas M. Gill ${ }^{1}$, \\ Tymon Barwicz ${ }^{1}$, Steven M. Shank ${ }^{4}$, Yurii A. Vlasov ${ }^{1}$, Richard M. Osgood, Jr. ${ }^{3}$, Roel Baets ${ }^{2}$, Gunther \\ Roelkens $^{2}$ \\ ${ }^{1}$ IBM Thomas J. Watson Research Center, 1101 Kitchawan Road, Yorktown Heights, New York 10598, USA. \\ ${ }^{2}$ Photonics Research Group, Department of Information Technology, Ghent University - imec, Ghent, Belgium. \\ ${ }^{3}$ Department of Electrical Engineering, Columbia University, New York, New York 10027, USA. \\ ${ }^{4}$ IBM Systems \& Technology Group, Microelectronics Division, 1000 River St., Essex Junction, Vermont 05452, USA. \\ wgreen@us.ibm.com
}

\begin{abstract}
A mid-infrared silicon nanophotonic integrated circuit platform can have broad impact upon environmental monitoring, personalized healthcare, and public safety applications. Development of various mid-IR components, including optical parametric amplifiers, sources, modulators, and detectors, is reviewed.

OCIS codes: (130.0130) Integrated optics; (300.6340) Spectroscopy, infrared
\end{abstract}

To date, the advancement of sophisticated photonic integrated circuits has largely been driven by ever-increasing demands for high bandwidth within long-haul and short-reach optical telecommunication networks, at reduced cost and low power consumption. However, the distinct advantages of the photonic integrated circuit concept, particularly the incorporation of advanced functionality into a small size and weight footprint, long-term reliability, and low-cost manufacturing, are equally significant to optical applications lying outside the telecom-band communications space.

The mid-infrared (mid-IR) spectrum, spanning wavelengths from approximately $2-14 \mu \mathrm{m}$, is highly relevant to a number of technological areas, including environmental monitoring, personalized results-driven healthcare, and public safety. This optical spectrum is often referred to as the "molecular fingerprinting" spectrum, as it includes the fundamental rovibrational absorption lines of many molecules of practical interest. Mid-IR spectroscopic sensors may be used to monitor the presence and concentration of chemical and/or biological molecular species within vapors, aerosols, or liquids, via their distinct rovibrational resonances [1]. Spectroscopy within the mid-IR is superior from a sensitivity standpoint, as the fundamental absorption lines can be 100-1000 times stronger than the higher-order overtone and combination lines present in the near-IR.

Contemporary mid-IR spectroscopic systems are based upon free-space bulk optical designs, whose considerable footprint, power consumption, and cost is prohibitive for distributed sensor networks. Therefore, the availability of highly integrated, compact mid-IR spectrometer sensors can have a significant technological impact [2-4]. For example, by spectroscopically quantifying the distribution of combustion-related atmospheric emissions over time [5], such devices can be used to optimize traffic patterns in urban areas, and to monitor fossil fuel-burning power plants on the electric grid. Furthermore, mid-IR spectrometers can provide a means for individuals and healthcare providers to promote healthier living, through non-invasive monitoring of bio-marker molecules in exhaled breath [6]. Finally, mid-IR spectroscopy is important in the homeland security sector, where it can be applied for detection of threats including toxic chemical/biological agents [7] and explosives residues [8].

In order to facilitate the above applications, our research targets a mid-IR photonic integrated circuit platform based upon silicon-on-insulator (SOI) nanophotonic waveguides. In principle, such waveguides can be designed to operate throughout silicon's broad mid-IR transparency spectrum (up to $\sim 8.5 \mu \mathrm{m}[2,4]$ ), moderated by the transmittance of the surrounding cladding materials. Our work has resulted in a toolkit of ultra-compact silicon midIR components, including novel optical parametric amplifiers, broadband sources, electrooptic modulators, filters, and detectors. Design concepts have made advantageous use of four-wave mixing (FWM), as this nonlinear optical process becomes highly efficient for mid-IR wavelengths near and longer than $2.2 \mu \mathrm{m}$, silicon's two-photon absorption threshold [9]. Moreover, the strong optical confinement characteristic of silicon nanophotonic waveguides can be leveraged to produce nonlinear optical interactions up to $10^{5}$ times larger than those in silica optical fibers [10]. 
Design of dispersion-engineered silicon nanophotonic waveguides has led to demonstration of the first silicon mid-IR optical parametric amplifier (OPA), which provided a net off-chip gain exceeding $13 \mathrm{~dB}$ near $\lambda=2.2 \mu \mathrm{m}$ [11]. Subsequently, reduction of propagation losses and an improved understanding of the FWM phase-matching conditions produced an OPA with an extremely broadband gain spectrum extending from 1.9-2.5 $\mu \mathrm{m}$, and parametric gain exceeding $50 \mathrm{~dB}$ [12]. This large gain has been applied to demonstrate several novel silicon mid-IR light sources, including a wavelength-tunable mid-IR optical parametric oscillator [13], and a supercontinuum source emitting from the telecom-band to the mid-IR [14]. Furthermore, free-carrier dispersion-based electrooptic Mach-Zehnder modulators have recently been characterized at mid-IR wavelengths near $2.2 \mu \mathrm{m}$, illustrating that design methodologies previously applied to telecom-band modulators can also be effective at longer wavelengths [15]. Finally, a mid-IR photodetection device which spectrally translates signals near $2.4 \mu \mathrm{m}$ to the telecom-band near $1.6 \mu \mathrm{m}$ with simultaneous amplification of $19 \mathrm{~dB}$, has been demonstrated [16]. This device can not only eliminate the bulky and power-hungry cryogenic cooling requirements traditionally associated with mid-IR photodetectors, but it can also operate with a significantly larger electronic signal-to-noise ratio. The above components may be integrated to perform the critical functions within a mid-IR integrated sensor circuit.

Our focus upon an SOI mid-IR photonics platform is underpinned by the availability of advanced silicon microelectronics industry manufacturing infrastructure, in addition to CMOS compatibility for optical sensors integrated with low-power CMOS interface electronics $[17,18]$. Nevertheless, a number of alternative waveguide substrates, including silicon-on-sapphire [19-21], silicon-on-porous-silicon [22], silicon-on-diamond [23], air-clad silicon membranes [24], silicon nitride [25, 26], germanium-on-silicon [4], chalcogenide glass [27], and fluoride crystal materials [28], can also be used for mid-IR photonic integrated circuit components. If desired, appropriate integration methodologies may be applied to combine the enabling features of several of these material systems.

\section{References}

[1] F. K. Tittel, D. Richter, and A. Fried, "Mid-Infrared Laser Applications in Spectroscopy," in Solid-State Mid-Infrared Laser Sources, I. T. Sorokina and K. L. Vodopyanov, eds. (Springer-Verlag, Berlin Heidelberg, 2003), pp. 445-516.

[2] R. A. Soref, S. J. Emelett, and W. R. Buchwald, "Silicon waveguided components for the long-wave infrared region," J. Opt. A - Pure Appl. Op. 8, 840-848 (2006).

[3] R. Soref, "Towards silicon-based longwave integrated optoelectronics (LIO)," SPIE Proc. 6898, paper 6898-6895 (2008).

[4] R. Soref, "Mid-infrared photonics in silicon and germanium," Nature Photon. 4(8), 495-497 (2010).

[5] U. Willer, M. Saraji, A. Khorsandi, P. Geiser, and W. Schade, "Near- and mid-infrared laser monitoring of industrial processes, environment and security applications," Opt. Laser Eng. 44(7), 699-710 (2006).

[6] K. Namjou, C. B. Roller, and P. J. McCann, "The Breathmeter: A new laser device to analyze your health," IEEE Circuits \& Devices Magazine, 2006, pp. 22-28.

[7] J. A. Seeley and J. M. Richardson, "Early warning chemical sensing," Lincoln Laboratory Journal 17(1), 85-99 (2007).

[8] R. Furstenberg, C. A. Kendziora, J. Stepnowski, S. V. Stepnowski, M. Rake, M. R. Papantonakis, V. Nguyen, G. K. Hubler, and R. A. McGill, "Stand-off detection of trace explosives via resonant infrared photothermal imaging," Appl. Phys. Lett. 93, 224103 (2008).

[9] A. D. Bristow, N. Rotenberg, and H. M. van Driel, "Two-photon absorption and Kerr coefficients of silicon for 850-2200 nm," Appl. Phys. Lett. 90, 191104 (2007).

[10] J. I. Dadap, N. C. Panoiu, X. Chen, I.-W. Hsieh, X. Liu, C.-Y. Chou, E. Dulkeith, S. J. McNab, F. Xia, W. M. J. Green, L. Sekaric, Y. A. Vlasov, and J. Osgood, R. M., "Nonlinear-optical phase modification in dispersion-engineered Si photonic wires," Opt. Express 16(2), 1280-1299 (2008).

[11] X. Liu, J. Osgood, R. M., Y. A. Vlasov, and W. M. J. Green, "Mid-infrared optical parametric amplifier using silicon nanophotonic waveguides," Nature Photon. 4(8), 557-560 (2010).

[12] B. Kuyken, X. Liu, G. Roelkens, R. Baets, J. Osgood, R. M., and W. M. J. Green, "50 dB parametric on-chip gain in silicon photonic wires," Opt. Lett. 36(22), 4401-4403 (2011).

[13] B. Kuyken, X. Liu, J. Osgood, R. M., R. Baets, G. Roelkens, and W. M. J. Green, "Widely tunable silicon mid-infrared optical parametric oscillator," presented at the Group IV Photonics, London, UK, 2011.

[14] B. Kuyken, X. Liu, J. Osgood, R. M., R. Baets, G. Roelkens, and W. M. J. Green, "Mid-infrared to telecom-band supercontinuum generation in highly nonlinear silicon-on-insulator wire waveguides," Opt. Express 19(21), 20172-20181 (2011).

[15] M. A. Van Camp, S. Assefa, D. M. Gill, T. Barwicz, S. M. Shank, Y. A. Vlasov, and W. M. J. Green, "Silicon nanophotonic mid-IR MachZehnder electrooptic modulator," presented at the Conference on Lasers and Electro-Optics, San Jose, USA, 2012.

[16] X. Liu, B. Kuyken, G. Roelkens, R. Baets, J. Osgood, R. M., and W. M. J. Green, "Bridging the mid-infrared-to-telecom gap with silicon nanophotonic spectral translation," Nature Photon. 6(10), 667-671 (2012).

[17] W. M. J. Green, S. Assefa, A. Rylyakov, C. L. Schow, F. Horst, and Y. A. Vlasov, "CMOS Integrated Silicon Nanophotonics: Enabling Technology for Exascale Computational Systems," presented at the SEMICON, Chiba, Japan, 1-3 December, 2010.

[18] S. Assefa, W. M. J. Green, A. Rylyakov, C. L. Schow, F. Horst, and Y. A. Vlasov, "CMOS Integrated Silicon Nanophotonics: Enabling Technology for Exascale Computational Systems," presented at the Optical Fiber Communications, Los Angeles, CA, 6-10 March 2011, 2011.

[19] T. Baehr-Jones, A. Spott, R. Ilic, B. Penkov, W. Asher, and M. Hochberg, "Silicon-on-sapphire integrated waveguides for the mid-infrared," Opt. Express 18(12), 12127-12135 (2010). 
[20] A. Spott, Y. Liu, T. Baehr-Jones, R. Ilic, and M. Hochberg, "Silicon waveguides and ring resonators at 5.5 mm," Appl. Phys. Lett. 97 , $213501(2010)$.

[21] F. Li, S. D. Jackson, C. Grillet, E. Magi, D. Hudson, S. J. Madden, Y. Moghe, C. O'Brien, A. Read, S. G. Duvall, P. Atanackovic, B. J. Eggleton, and D. J. Moss, "Low propagation loss silicon-on-sapphire waveguides for the mid-infrared," Opt. Express 19(16), 15212-15220 (2011).

[22] G. Z. Mashanovich, M. M. Milosevic, M. Nedeljkovic, N. Owens, B. Xiong, E. J. Teo, and Y. Hu, "Low loss silicon waveguides for the mid-infrared," Opt. Express 19(8), 7112-7119 (2011).

[23] D. Liang, M. Fiorentino, S. T. Todd, G. Kurcveil, R. G. Beausoleil, and J. E. Bowers, "Fabrication of silicon-on-diamond substrate and lowloss optical waveguides," IEEE Photonics Technol. Lett. 23(10), 657-659 (2011).

[24] R. Shankar, R. Leijssen, I. Bulu, and M. Loncar, "Mid-infrared photonic crystal cavities in silicon," Opt. Express 19(6), 5579-5586 (2011).

[25] J. S. Levy, A. Gondarenko, M. A. Foster, A. C. Turner-Foster, A. L. Gaeta, and M. Lipson, "CMOS-compatible multiple-wavelength oscillator for on-chip optical interconnects," Nature Photon. 4(1), 37-40 (2010).

[26] Y. Yue, L. Zhang, H. Huang, R. G. Beausoleil, and A. E. Willner, "Silicon-on-nitride waveguide with ultralow dispersion over an octavespanning mid-infrared wavelength range," IEEE Photonics J. 4(1), 126-132 (2012).

[27] X. Gai, D.-Y. Choi, S. Madden, Z. Yang, R. Wang, and B. Luther-Davies, "Supercontinuum generation in the mid-infrared from a dispersion-engineered As2S3 glass rib waveguide," Opt. Lett. 37(18), 3870-3872 (2012).

[28] C. Y. Wang, T. Herr, P. Del'Haye, A. Schliesser, J. Hofer, R. Holzwarth, T. W. Hansch, N. Picque, and T. J. Kippenberg, "Mid-infrared optical frequency combs based on crystalline microresonators," arXiv:1109.2716v2 (2012). 